\title{
Affaissements miniers tardifs et feux de vieux travaux à Saint-Étienne
}

\section{F. BARDOT}

Expert agréé par la Cour de cassation, Lyon

\section{P. DUFFAUT}

Vice-Président de «Espace souterrain »,

Paris

\section{G. SANGLERAT}

Expert agréé par la Cour de cassation, Lyon
La ruine des bâtiments du «Petit Clos », sur la colline de Côte Chaude à Saint-Étienne, est due à des affaissements miniers tardifs, plus d'un siècle après l'exploitation de couches situées à moins de 100 mètres de profondeur. Une autre caractéristique remarquable du cas présenté est la combustion du charbon dans la zone des vides abandonnés. Si les affaissements au-dessus de cavités sont relativement classiques, même en I'absence d'exploitation de mines ou de carrières, le feu est spécifique du charbon. Les mines en activité en ont l'expérience, mais les feux de "vieux travaux» sont pour le moins méconnus.

\section{Late mine subsidence and fires inside old workings in Saint-Étienne}

The decay of Petit Clos housing estate, situated on Cóte Chaude hill in Saint-Etienne, is due to late mining subsidence, a century after the exploitation of coal layers within $100 \mathrm{~m}$ depth. Another remarkable feature of the case is the combustion of coal inside mine cavities. Whereas subsidence is rather common above any cavities, even above non mining ones, fires are specific to coal. Working coal mines have experience with fires, however fires inside old workings have remained little known. 


\section{Introduction}

La ville de Saint-Étienne est née des mines de charbon, exploitées dès le XIII siècle à partir des affleurements, puis de plus en plus profondément, jusqu'à leur fermeture progressive de 1950 à 1975. Comme témoins de cette longue période, il reste les célèbres terrils qui ajoutent leurs collines coniques aux nombreux reliefs naturels, et aussi des noms de lieux évocateurs, tel celui de Côte Chaude.

A Côte Chaude les Houillères de la Loire ont libéré en 1969 un vaste périmètre transformé en Zone d'aménagement concerté avec l'accord de la Ville et de la Direction départementale de l'Équipement. La ZAC du Grand Clos ( 80 hectares) reçoit un plan d'urbanisme, les voies et réseaux sont en place, des sociétés d'HLM achètent des lots, l'une d'elles construit trois immeubles en 1980 comportant 59 logements (cf. Fig. 1).

Les premiers logements sont habités aussitôt. Mais des désordres apparaissent puis s'aggravent (cf. Fig. 2 à 6), au point que les immeubles doivent être évacués (arrêté de péril en juin 1983), et plus tard démolis. Depuis, la totalité de la ZAC reste en friche.

Comment en est-on arrivé là? Les faits, les principales interventions et les diverses tentatives d'explication sont rappelés ci-dessous, en insistant sur les séquelles des travaux miniers: non seulement affaissements de la surface, mais aussi feux de mine, spécifiques des mines de charbon.
Guy Sanglerat, en tant que conseil du maître d'ouvrage, Francis Bardot et Pierre Duffaut, en tant qu'experts judiciaires, sont intervenus dans le cadre d'une procédure d'appel (les aspects judiciaires ne sont pas analysés ci-dessous; ils feront l'objet d'une autre communication).

\section{2}

\section{Rappel des faits}

Des nombreux puits d'exploitation ouverts au cours des siècles sur la colline de Côte Chaude, les deux derniers, le puits Palluat et le puits des Rosiers, ont été régulièrement fermés en 1899, l'exploitation des couches profondes se poursuivant à partir de puits plus lointains. Le site reste occupé par des bureaux et ateliers divers, cependant qu'à l'est une cité d'habitation pour les mineurs est construite, rue de Chavassieux, vers 1950 ; elle comporte huit immeubles de deux à quatre niveaux dont l'implantation est indiquée sur la figure 7 .

Après le départ des Houillères, l'aménagement de la ZAC a commencé par les voiries centrales, les rues des Houillères et Louise-Michel dès 1975; le boulevard Rhin et Danube, qui limite la ZAC à l'ouest, n'a été terminé qứen 1981. Les lots viabilisés sont mis en vente avec la clause habituelle d'exonération de responsabilité de l'exploitant minier: le terrain est pris en l'état par l'acheteur, sans recours contre le vendeur en cas de vice du sol attribuable aux exploitations passées.

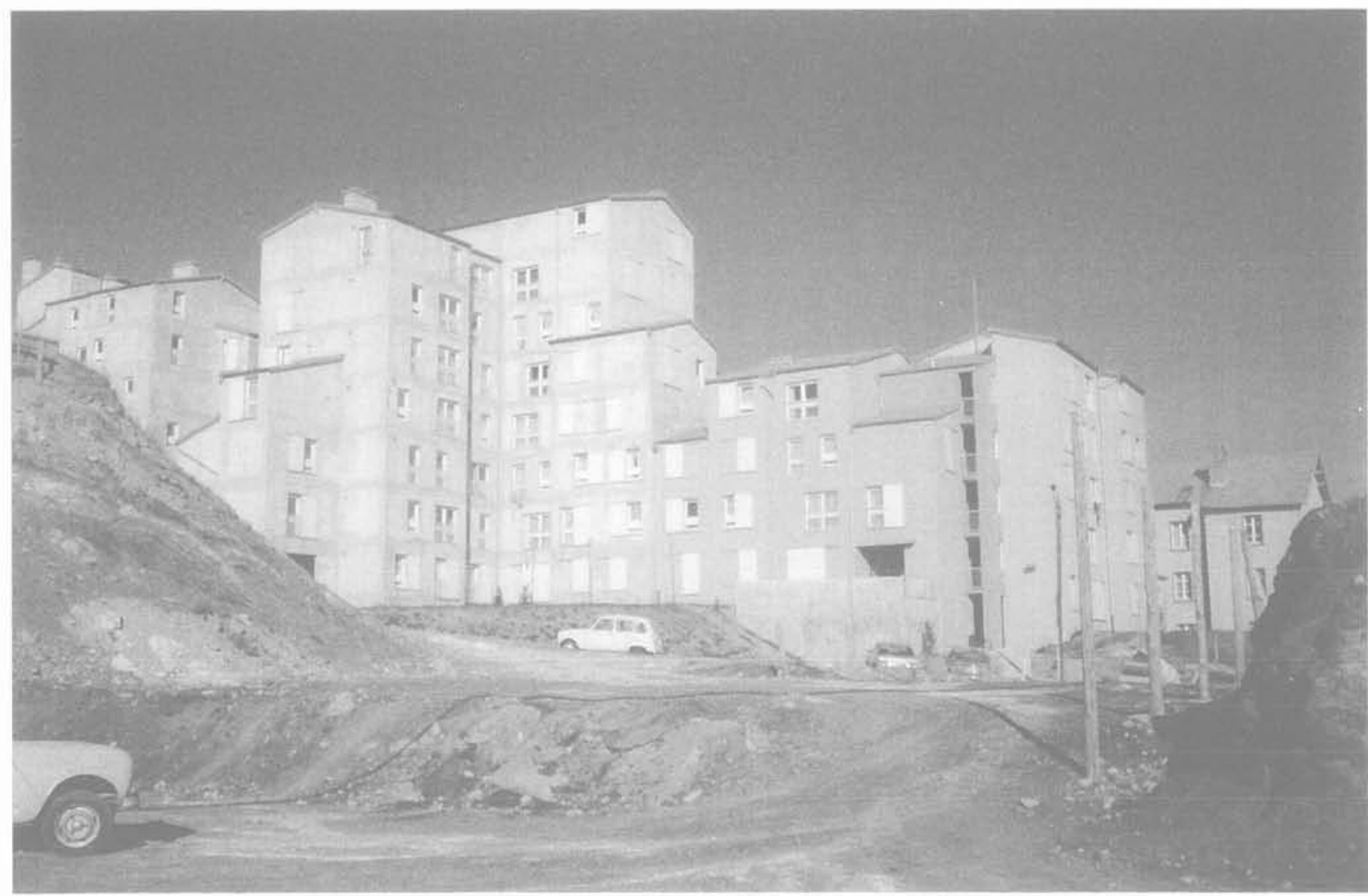

FIG.1 Vue d'ensemble des bâtiments du Petit Clos sinistrés. Au premier plan fouille de pleine masse de l'opération « Vallée de l'Ondaine $》$.

Overview of «Petit Clos» damaged structures. Forefront, excavation of «Vallée de l'Ondaine» operation. 


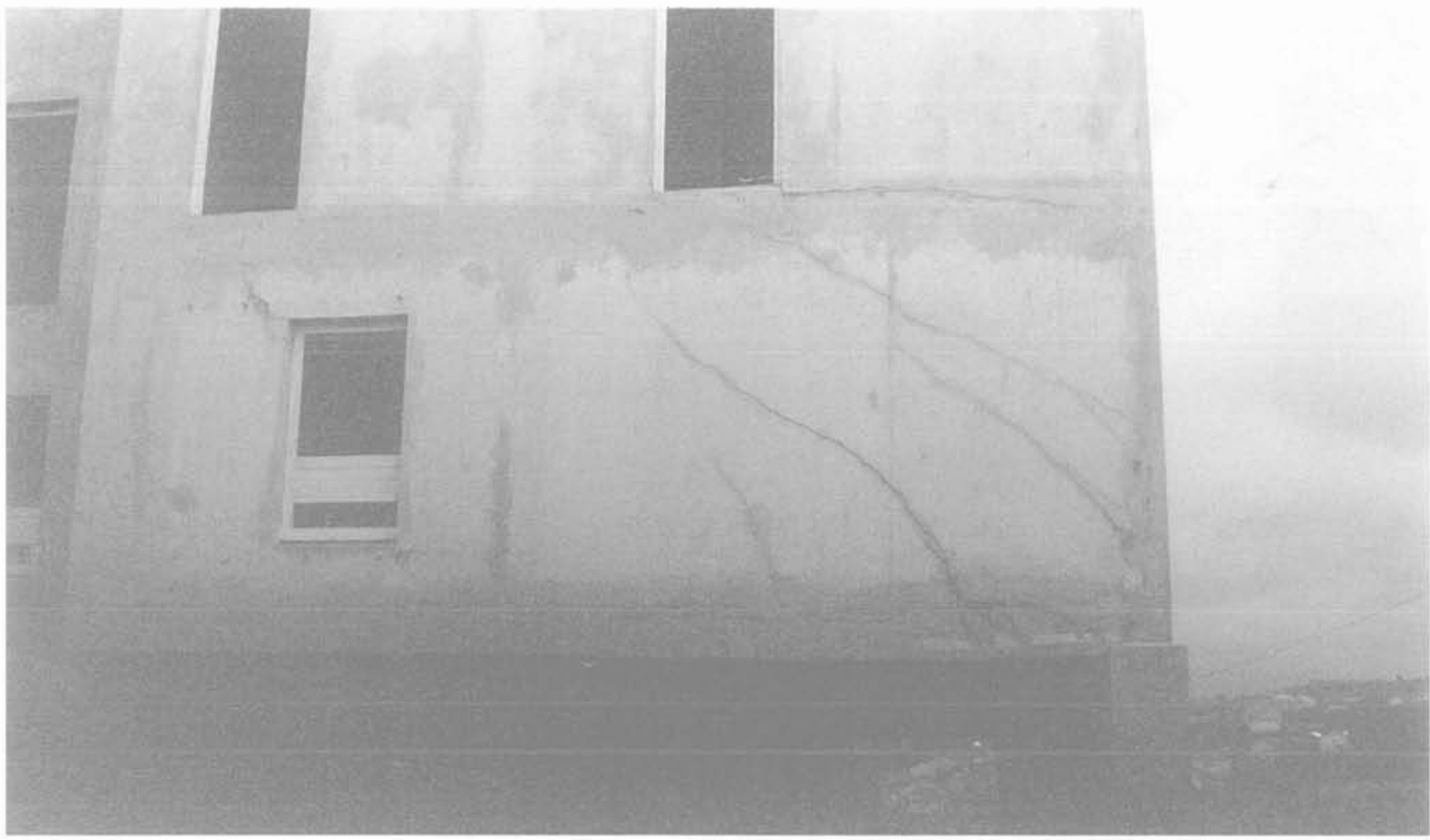

fiG.2 Fissures caractéristiques de tassements différentiels. Typical cracks due to differential settlements.

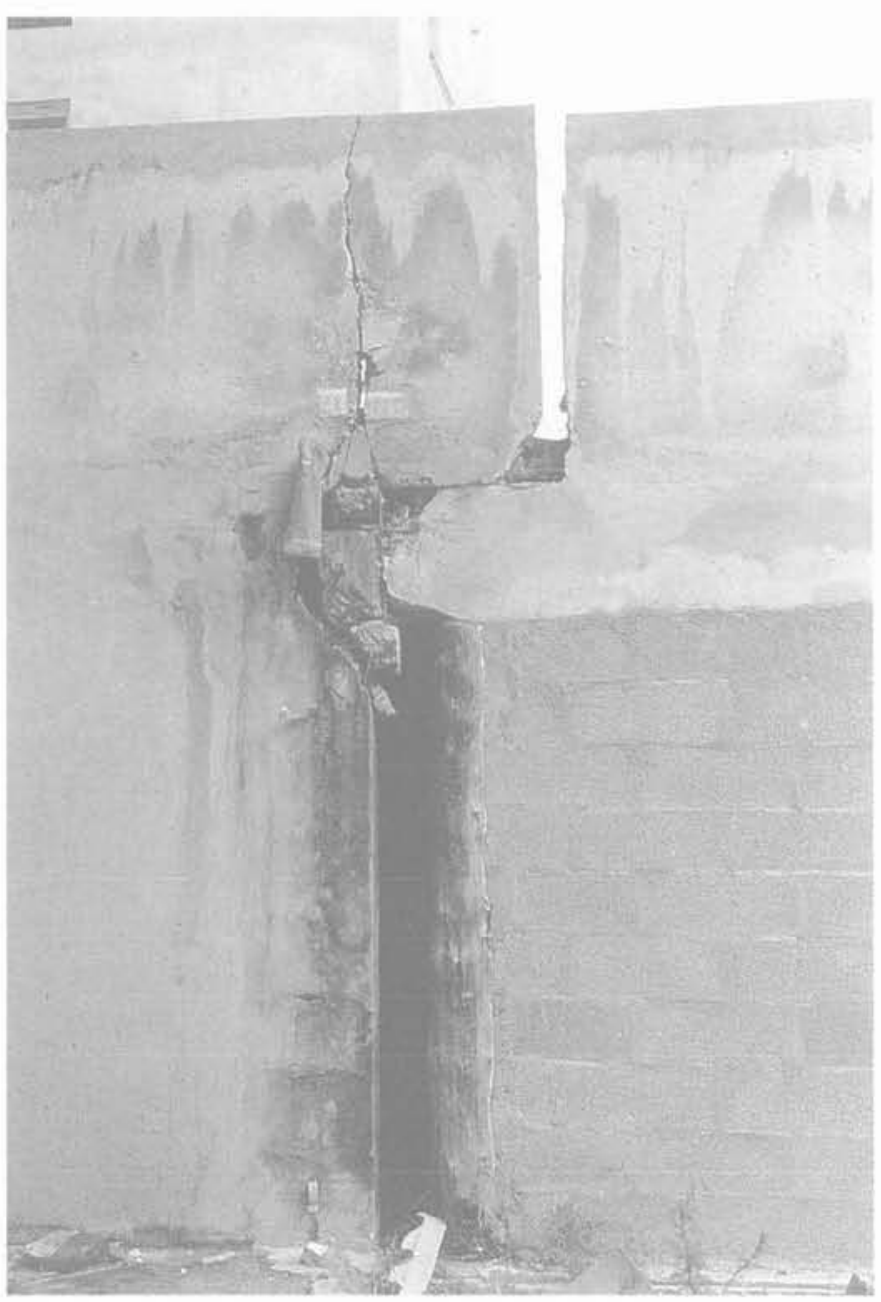

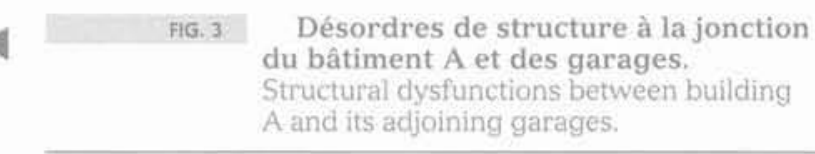

Deux parcelles sont vendues, le Petit Clos et Vallée de l'Ondaine. Sur la première $\left(4100 \mathrm{~m}^{2}\right)$ une étude de sol est réalisée en 1975 , basée sur trois sondages carottés de 10, 30 et $31 \mathrm{~m}$, et deux douzaines d'essais au pénétromètre dynamique Sermes, limités à une profondeur de $3 \mathrm{~m}$ maximum par la présence du rocher.

Après un changement de propriétaire et de projet, trois immeubles cruciformes de quatre à sept niveaux sont construits, de 1979 à 1980, sur la parcelle du Petit Clos (cf. Fig. 7). Ils seront livrés successivement:

C: en décembre 1979;

B: en mai 1980;

A : en septembre 1980 .

La structure des bâtiments est constituée par des refends porteurs perpendiculaires aux façades. Dès mai 1980 le bâtiment C présente des fissures, attribuées tout d'abord à des retraits, puis à des chocs thermiques ou à des malfaçons; elles vont s'aggraver et gagner les deux autres bâtiments; en septembre 1981, sur les 59 appartements construits, 46 ont été occupés dont 7 ont dû déjà être évacués; les fissures affectent non seulement les façades mais aussi les structures porteuses (cf. Fig. 2, 3 et 4), d'autant que les immeubles contigus s'inclinent l'un vers l'autre (cf. Fig. 5).

Afin de mieux connaître les caractéristiques des couches porteuses, le maitre d'ouvrage fait exécuter par Bachy deux sondages carottés profonds $(63 \mathrm{~m})$, qui mettent en évidence des vides et des décompressions que les premiers sondages n'avaient pas décelés. 


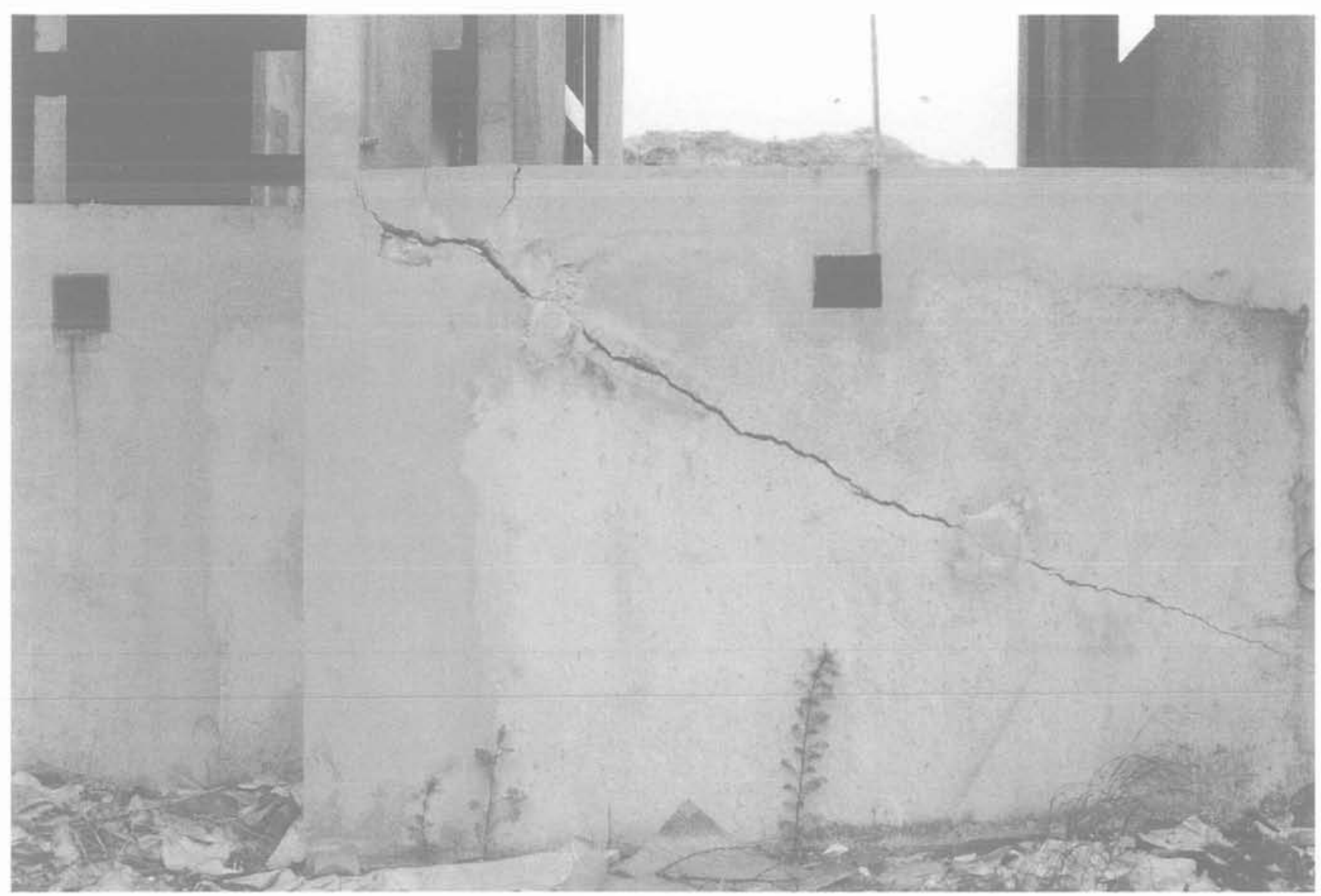

FIG.4 Désordres dans les soubassements.

Damages in the basements.

Sur l'autre parcelle vendue, le même entrepreneur de maçonnerie qui avait construit le Petit Clos allait commencer, au début de 1981, la construction de 121 logements pour la SA d'HLM "Vallée de l'Ondaine m. Il s'inquiète des désordres survenus au Petit Clos et appelle en consultation en avril 1981 Guy Sanglerat qui impute formellement la fissuration des immeubles à des tassements différentiels (ce qui n'était alors qu'une des hypothèses émises en janvier par certains experts). Le rapport Bachy du 4 juin 1981 attribue les désordres du Petit Clos à des vides d'origine minière et émet pour la première fois l'hypothèse de feux souterrains (cf. Fig. 15).

Comme les premiers terrassements sur la parcelle Vallée de r'Ondaine ont déclenché des fumerolles, de nouveaux sondages sont entrepris, où sont mesurées des températures très élevées, jusqu'à $240^{\circ} \mathrm{C}$ ! (cf. Fig. 8). Le projet de cette nouvelle construction est alors abandonné.

Il est à noter qu'une température dépassant $280^{\circ} \mathrm{C}$ a été enregistrée dans un sondage effectué sur le terrain du Petit Clos.

Nous disons qu'au droit du terrain du Petit Clos, la température à $60 \mathrm{~m}$ de profondeur a dépassé $280^{\circ} \mathrm{C}$. car le thermomètre a fondu à cette température, on ignore donc la température maximale réelle atteinte à ce niveau.

Au Petit Clos, des mesures sont enfin entreprises: contrôle des pentes des planchers et des faux aplombs des murs, nivellement au large des immeubles (voir §4). Diverses réparations sont envisagées, notamment pour désolidariser les immeubles A et B (cf. Fig. 5 et 6), et un programme de remplissage des vides sousjacents par injection au coulis de ciment est mis en œuvre par Bachy à l'automne sur recommandation d'un rapport du Laboratoire Central des Ponts et Chaussées (14 septembre 1981).

Les Houillères procèdent à des embouages (voir \$ 5.1) en 1980-1981 sur leurs terrains et en 1981 sur celui du Petit Clos.

A la même époque les terrassements du boulevard Rhin et Danube ont aussi déclenché ou aggravé des fumerolles. De nouvelles campagnes d'embouage des $\mathrm{HBCM}^{(1)}$ ont tenté de les réduire en avril 1982 et avrilmai 1983 (une zone non aedificandi avait été réservée dès 1974 en raison de ces manifestations, sur une largeur de $50 \mathrm{~m}$ le long du boulevard).

Ces travaux d'embouage devaient, en principe, d'une part consolider le terrain des HBCM servant d'assise à la route principale prévue pour desservir la $Z A C$, et d'autre part abaisser les très hautes températures résultant de la combustion des couches profondes de charbon et des échauffements consécutifs observés dans les volumes voisins.

Ces embouages étaient réalisés par forages de trous verticaux dans lesquels était injectée une boue extrêmement liquide avec cendres volantes. L'eau injectée suivait donc, tout naturellement, la pente descendante vers l'est de la « troisième Beaubrun » (cf. §3.1). Or, il a

(1) Houillères des Bassins du Centre Midi, branche régionale de Charbonnages de France, dont font partie les Houillères de la Loire. 


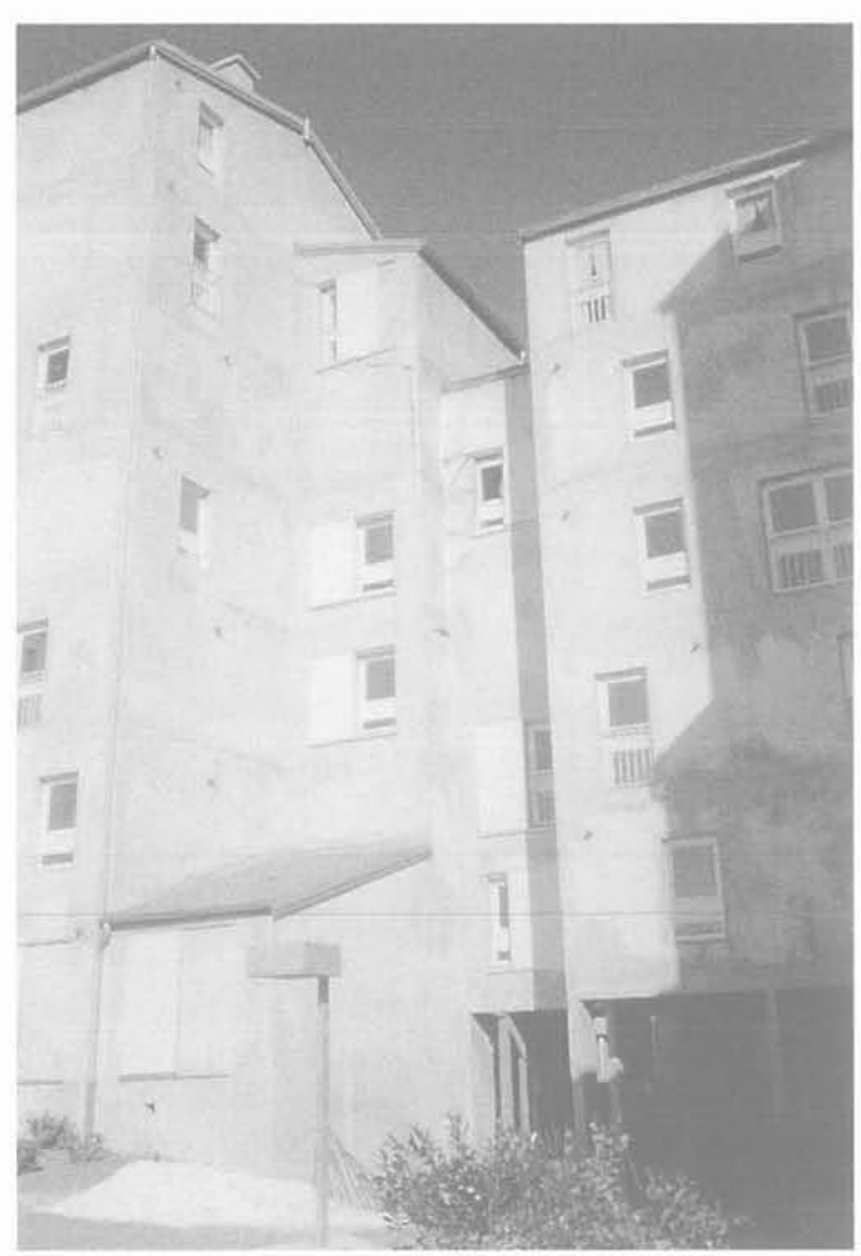

été constaté (cf. \$ 4.2 et Fig. 9) une aggravation brutale des tassements concomitante des embouages, notamment ceux de mai-juin 1983.

On peut donc admettre que ce faisant, cette eau, en très grande quantité, avait une quadruple action néfaste: - entraînement des fines, ce qui déséquilibre les blocs encastrés dans ces éléments:

- ramollissement et désagrégation du toit des “chambres» (cf. $\S 3.3$ et Fig. 14) susceptibles d'entraîner leur effondrement;

- chocs thermiques dans les piliers de charbon et scories réduisant leur force portante (jeter de l'eau froide sur un rocher préalablement réchauffé constituait autrefois une méthode d'abattage) :

- désagrégation des piliers de charbon ayant été transformés préalablement en scories.

Ces quantités d'eau ont donc pu déplacer vers l'aval des matériaux, ce qui a entraîné de nouveaux effondrements miniers à grande profondeur.

Les paragraphes suivants décrivent d'abord les terrains avec les (vieux travaux ) miniers, puis les affaissements observés et leur interprétation, enfin les feux de mine, principale originalité de cette affaire.

FG. 5 Jonction des bâtiments A et B fortement déformée.

Severely distorted joints between structures A and $\mathrm{B}$.

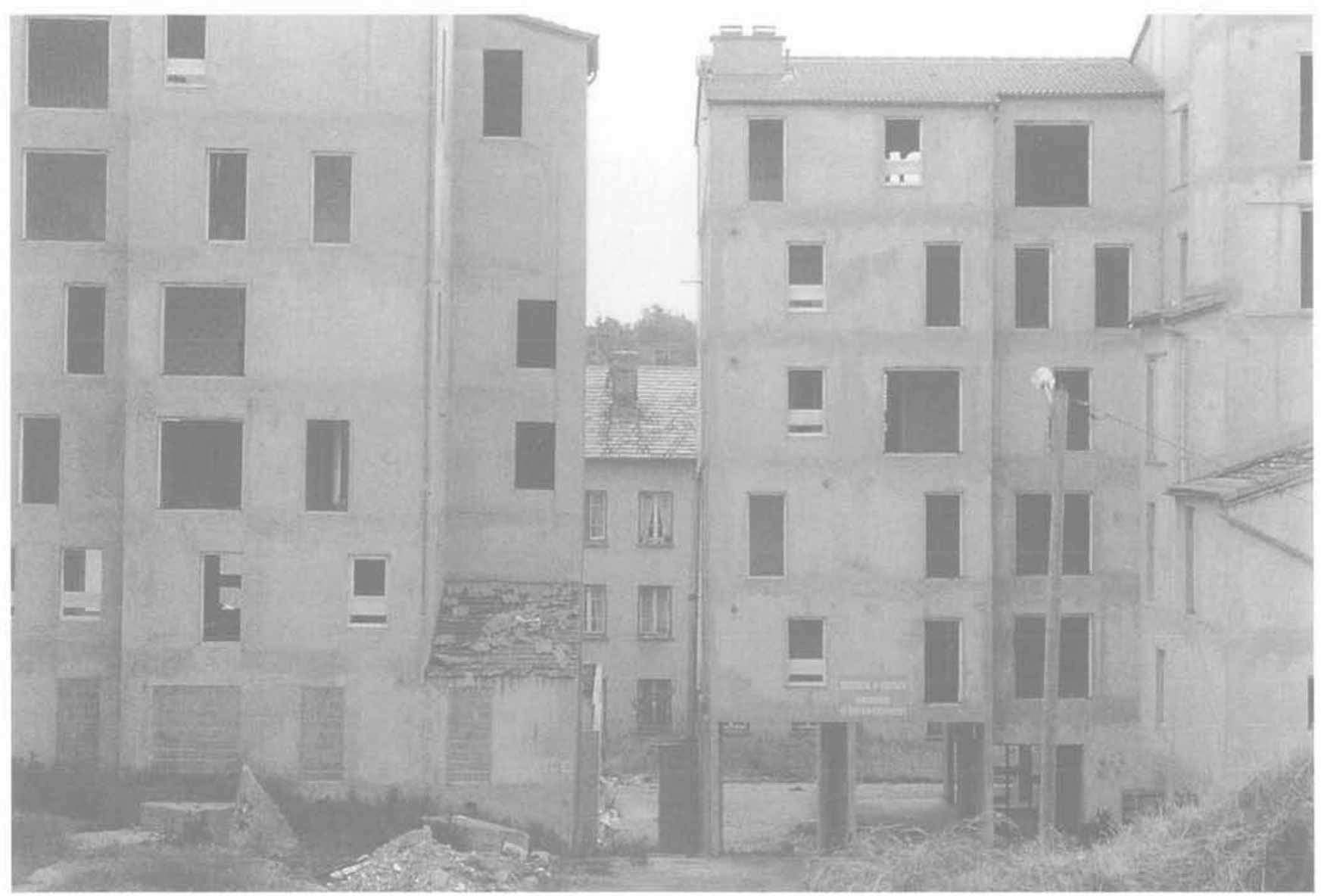

FG.6 Après la démolition de la jonction des bâtiments $A$ et $B$. Buildings $\mathrm{A}$ and $\mathrm{B}$ after the removal of their junction. 


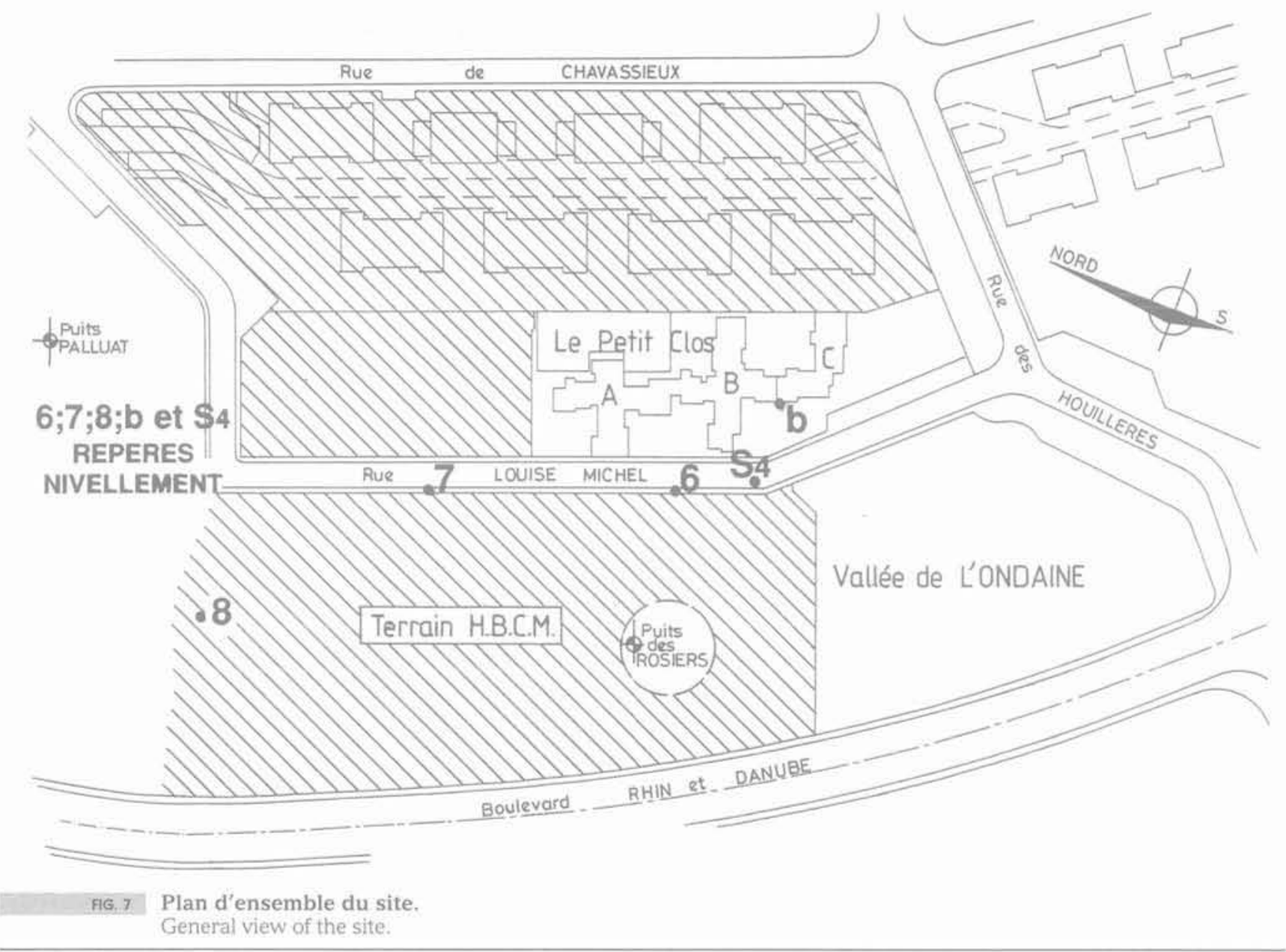

\section{3}

\section{Aperçu de la structure géologique couches de charbon et vieux travaux}

\section{1}

\section{Généralités}

Les terrains qui forment le substratum de la ville de Saint-Étienne appartiennent à l'étage Stéphanien. Ils comportent des alternances de grès et de schistes, avec de nombreuses couches de charbon. La description en a été donnée par Grüner, qui fut directeur de l'École des mines (une carte a été dessinée en 1857 et un atlas de 24 planches en 1882). Les couches de charbon ont été groupées en « faisceaux») et numérotées à partir de la surface; c'est le faisceau des "couches Beaubrun») (cf. Fig. 8 et 14) qui était exploité sous la colline de Côte Chaude.

A la différence des bassins houillers étendus, où des couches régulières peuvent se suivre sur des dizaines de kilomètres ou davantage, le bassin de Saint-Étienne présente de nombeuses irrégularités, dûment signalées par les auteurs:

- le schiste passe à la houille, réciproquement les couches de charbon contiennent des îlots stériles appelés « coufflées 》;

- la puissance ${ }^{221}$ varie de quelques centimètres à $10 \mathrm{ou}$ 15 mètres;

(2) Puissance $=$ épaisseur.
- les couches puissantes se subdivisent, ce qui rend leur numérotation imprécise;

- la pente des épontes ${ }^{(3)}$ est extrêmement changeante.

Cette complexité stratigraphique, faite de passages latéraux, de variations d'épaisseur et de pendage, et même de bifurcations, est fréquente dans les bassins houillers du Massif central, oủ elle témoigne d'une sédimentation deltaïque très irrégulière dans des lacs peu étendus; mais elle ne facilite pas l'exploitation. En effet, les méthodes doivent s'adapter aux épaisseurs et aux pendages, et le dépilage contourne les îlots stériles. Et cette complexité rend difficile aussi l'interprétation des plans de vieux travaux, où même l'attribution à telle ou telle couche dûment numérotée peut être remise en question.

\section{2}

\section{Les anciennes méthodes d'exploitation}

Commencée à ciel ouvert là où affleurait le charbon. l'exploitation s'est poursuivie en souterrain, bien avant la mise au point des méthodes modernes. Le meilleur rendement était obtenu "par éboulement, le piqueur profitant du git, ou clivage, pour obtenir de grosses tombées » (Leseure, 1901). Outre le risque couru par le mineur, cette méthode, poursuivie encore au début du $\mathrm{XIX}^{\mathrm{C}}$ siècle bien qu'interdite par l'administration, provoquait en surface des « crevasses immenses et des affais-

17in Les épontes sont des couches de terrains stériles encadrant le charbon. 


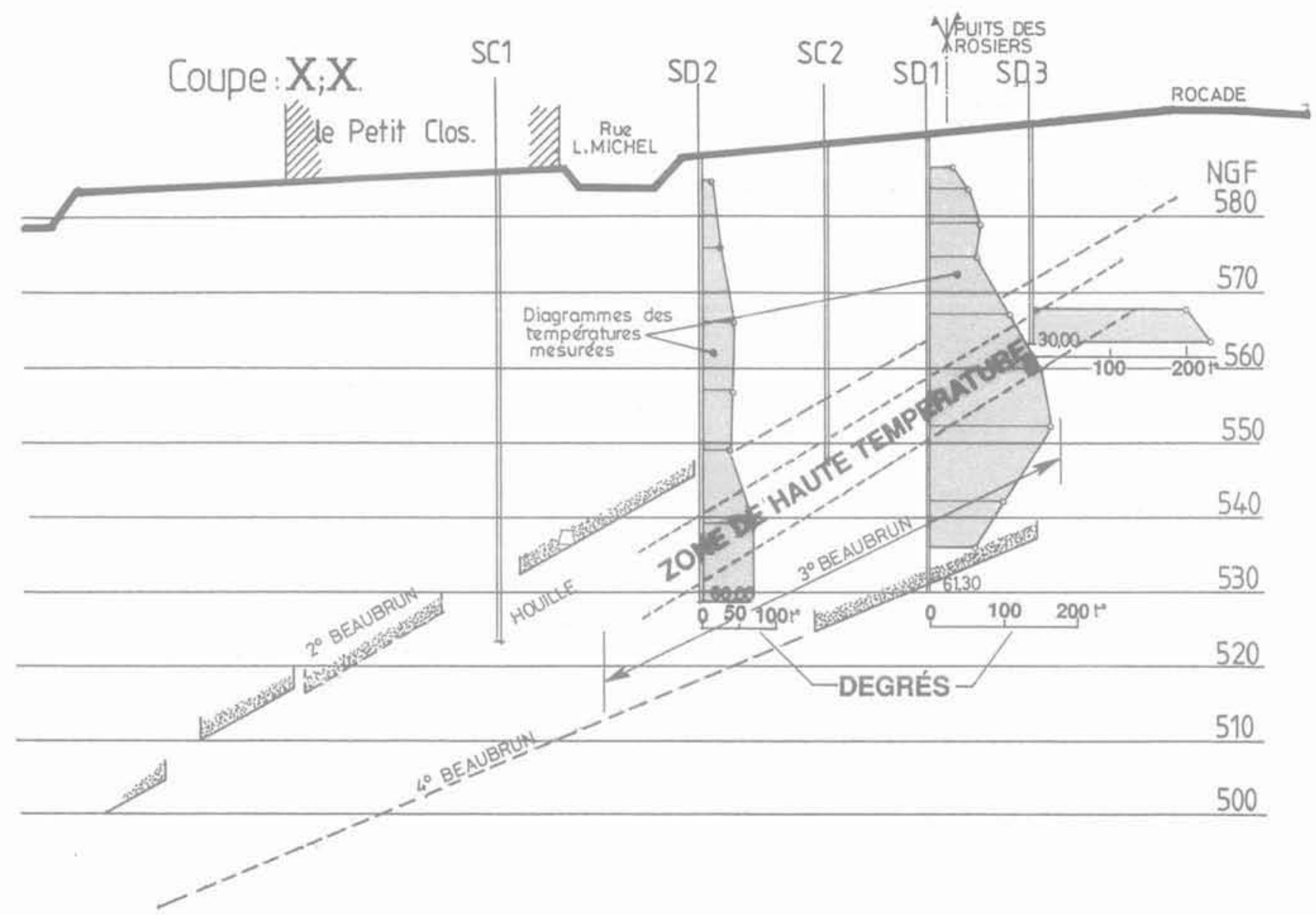

Coupe de la colline de Côte Chaude, couches de charbon et hautes températures (parcelle Vallée de I'Ondaine).

Section of Côte Chaude Hill, coal layers and high temperatures.

sements énormes y) (Simonin, 1867), et laissait en place au moins un quart du charbon et, même, la moitié dans les couches les plus épaisses. Les crevasses favorisaient l'inondation des chantiers par les eaux superficielles et le charbon en partie foisonné était sujet aux feux de mine.

Ensuite est venue une méthode dite par «chambres et piliers $x$ (cf. Fig. 14), avec reprise partielle des piliers et foudroyage. Le foudroyage moderne, associé à l'exploitation par tailles n'a été introduit qu'en 1937.

Un cours de Vidal (1961) confirme que toutes ces méthodes laissent dans le terrain des vides propices aux feux de mine, "vides (qui) peuvent se maintenir pendant des siècles (mais dont) l'éboulement se produira un jour, inopinément (voir d'autres citations dans le paragraphe 5 sur les feux).

\section{3}

\section{Les «vieux travaux " à Côte Chaude}

On entend par «vieux travaux » les traces d'exploitations anciennes dont ni la localisation ni les méthodes employées ne sont bien connues; ce sont des cicatrices indélébiles, souvent gênantes pour les travaux à venir, mais aussi de précieux témoins de méthodes disparues. C'est ici le premier aspect qui domine. Ainsi à Côte Chaude, les couches 2 et 3 ont été exploitées il y a plus de 150 ans.
L'ensemble des plans anciens est conservé par les Houillères (pour le compte de l'administration de tutuelle), mais ces plans sont difficiles à lire et à raccorder les uns aux autres. L'établissement de coupes verticales à partir des coupes des puits et des plans successifs des exploitations minières a été effectué en 1988 (cf. Fig. 8 et 12).

La complexité stratigraphique rend ce travail difficile et parfois incertain; si le pendage, qui va jouer un grand rôle dans la recherche du vide générateur de l'affaissement, n'est pas discuté, la présence et la position exacte d'une ou deux failles sont moins assurées et il subsiste une ambiguïté entre les deuxième et trojsième Beaubrun sous le terrain du litige. La figure 14 mentionne à faible profondeur la galerie Rambaud, qui a été fermée après une visite contradictoire, en juin 1981, au cours de la première expertise judiciaire.

\section{4}

\section{Les affaissements}

\section{1}

\section{Généralités}

Toute exploitation minière étendue se traduit par un affaissement généralisé de la surface. L'affaissement 


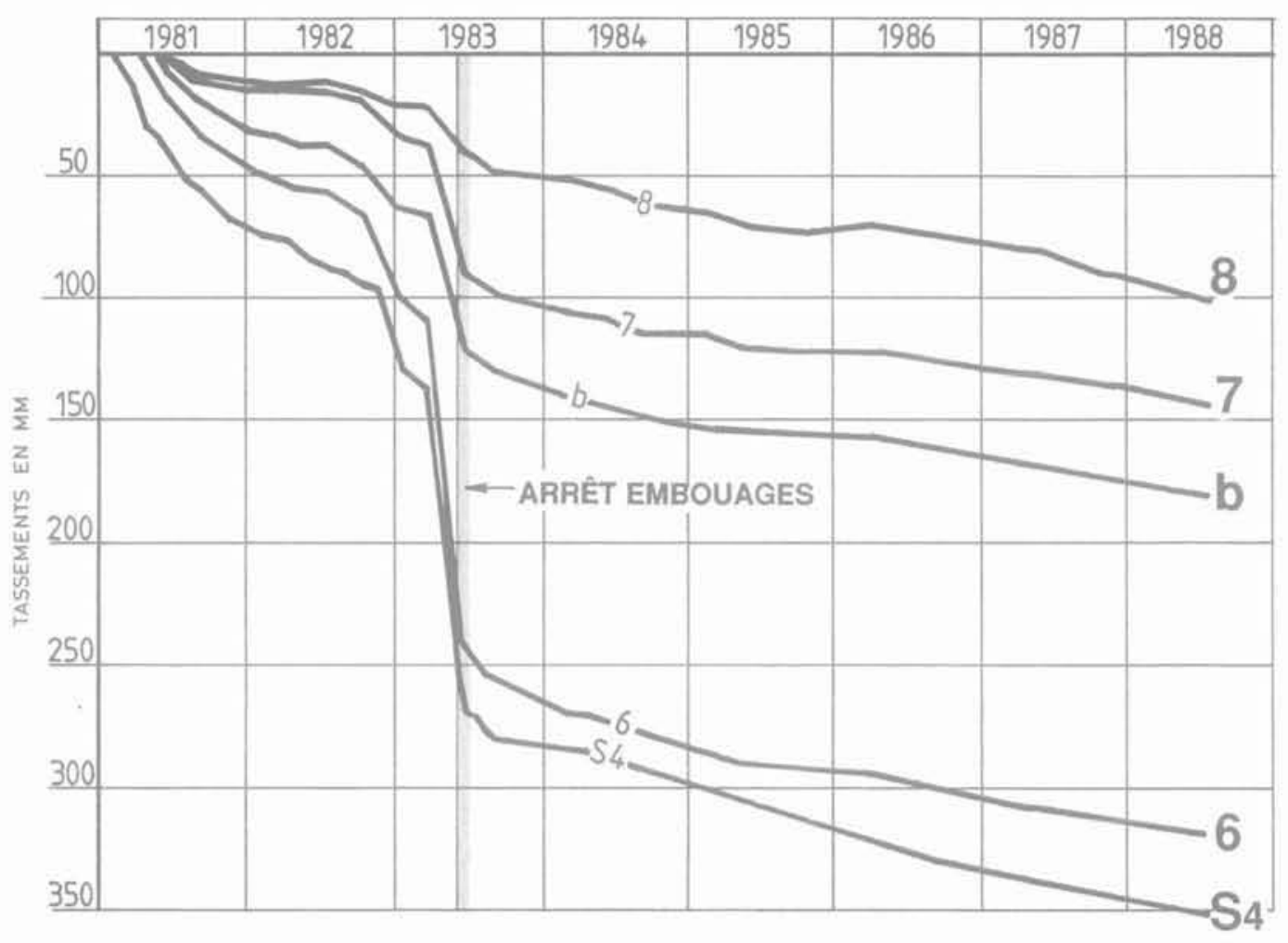

FG. 9 Tassements mesurés entre 1981 et 1988 pour les points $6,7,8$ b et $S_{4}$. Settlements on site between 1981 and 1988.

peut suivre immédiatement le tir si la couche exploitée est peu profonde (par exemple les carrières de gypse sous la forêt de Montmorency) ou plus souvent ne se répercuter à la surface qu'après un certain délai. Pour éviter ou réduire les affaissements en surface, il faut limiter l'exploitation et abandonner des piliers conséquents, appelés «stots de protection » (on protège ainsi les villages, les cours d'eau, les voies de communication).

Des affaissements brutaux, susceptibles de produire des effets destructeurs analogues à ceux des séismes, affectent les exploitations par "chambres et piliers», lorsqu'un grand nombre de ceux-ci s'écrasent simultanément. Ces affaissements, dits " effondrements spontanés $»$ et bien décrits en Lorraine, ont été observés aussi au-dessus de carrières, y compris en région parisienne.

Ainsi, un groupe d'habitations a été détruit à Clamart en 1961. Une liste a été publiée par Josien en 1995. Moins brutal, l'affaissement tardif du «stot » de Crusnes en 1977 a néanmoins fissuré les maisons qu'il aurait dû protéger (mines de fer de Lorraine).

\section{Nivellements (cf. Fig. 9)}

Lorsque le basculement l'un vers l'autre des immeubles du Petit Clos a été constaté, les premières mesures ont porté sur les faux aplombs des murs et l'inclinaison des planchers; dès janvier 1981, le pignon est du bâtiment B montrait un faux aplomb de $5 \mathrm{~cm}$ sur
$2,2 \mathrm{~m}$ et le nivellement des planchers des différences de $20 \mathrm{~cm}$ sur les $30 \mathrm{~m}$ du battiment $\mathrm{A}$, de $30 \mathrm{~cm}$ en sens inverse sur l'ensemble des deux autres ( $40 \mathrm{~m}$ environ).

Ces mesures mettent en évidence un affaissement minimal au centre de $30 \mathrm{~cm}$ auquel il faut ajouter l'affaissement inconnu aux extrémités et qu'il faudra ajouter aux mesures ultérieures.

Les Houillères ont un réseau de nivellement qui couvre le quartier: elles ont communiqué les courbes de la figure 9, à partir d'avril 1981 pour le point 6, le plus proche des bâtiments en cause, puis à partir de fin mai pour d'autres points dont les points 7,8 , et b; les points 6 et 7 étant sur le trottoir de l'avenue LouiseMichel et le point b sur le bâtiment C à la jonction du bâtiment B. Depuis janvier 1981, de son côté, le maitre d'ouvrage du Petit Clos avait fait niveler nombre d'autres points. L'ensemble de ces mesures a permis de tracer des courbes d'égal affaissement (cf. Fig. 10 et 11).

L'examen des courbes des tassements en fonction du temps de la figure 9 montre quatre phases successives:

a) ralentissement pendant 15 mois, pour un total de 89 , $60,40,15$ et $10 \mathrm{~mm}$ respectivement aux points $\mathrm{S}, 6, \mathrm{~b}, 7$ et 8: ce ralentissement pouvant être imputable aux injections Bachy

b) reprise nette puis faible ralentissement, sur 8 mois (pour atteindre respectivement 132, 115, 70, 40 et $22 \mathrm{~mm}$, dans le même ordre);

c) reprise très forte (sans précédent mesuré) en avrilmai 1983, pour atteindre respectivement 262, 243, 120, 90 et $50 \mathrm{~mm}$. 


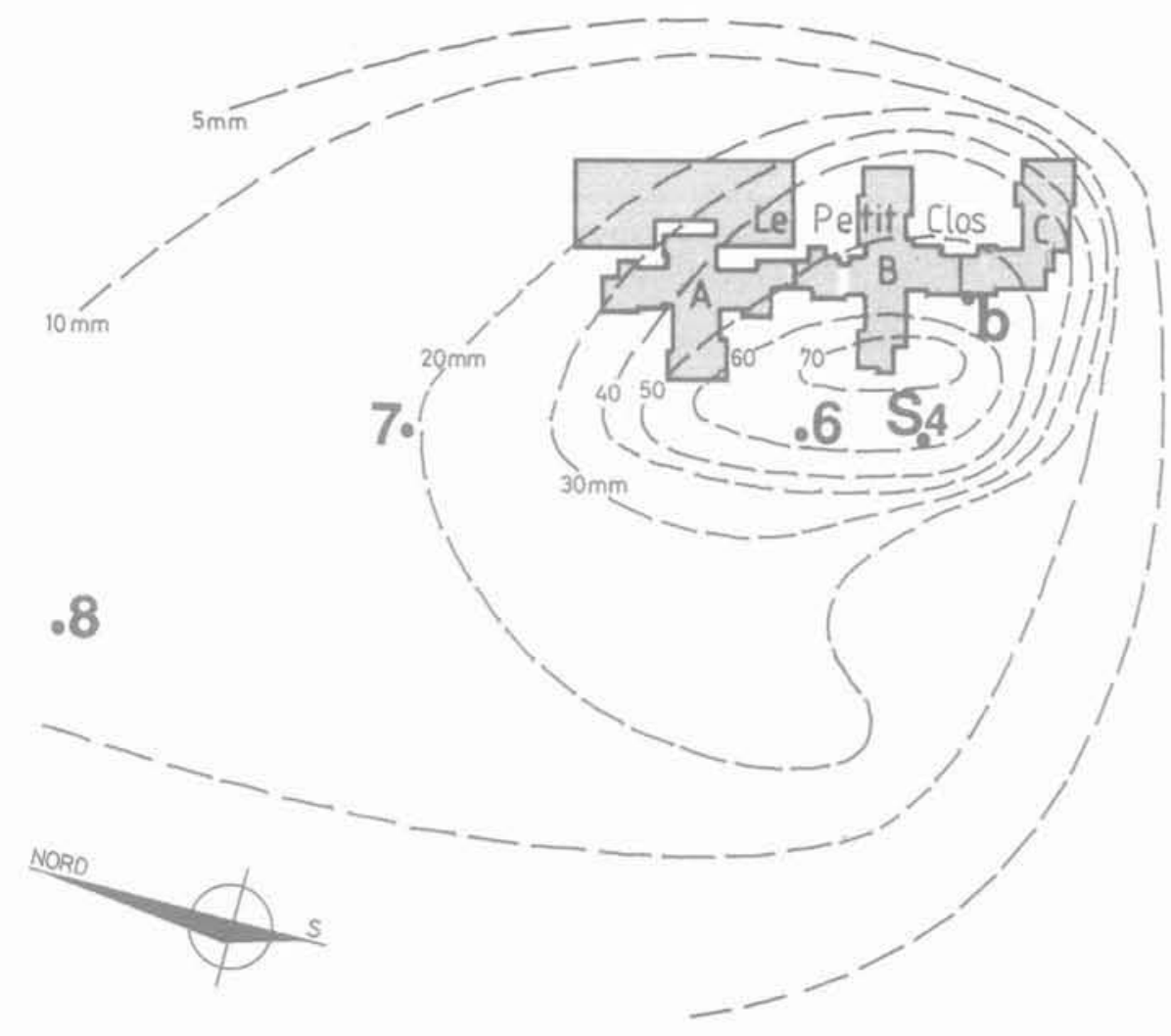

FIG 10 Courbes d'iso-tassements entre avril 1981 et octobre 1982. iso-settlements curves between April 1981 and October 1988.

L'accélération des tassements de surface entre décembre 1982 et mai 1983 a été provoquée par les embouages réalisés par les HBCM sur leur terrain. L'expert du TGI de Saint-Étienne, Georges Filliat, a donc eu tout à fait raison de mettre en demeure, le 14 mai 1983, les HBCM de suspendre ces travaux d'embouage qui, loin d'améliorer la stabilité du site, ont, au contraire, contribué à aggraver les affaissements miniers et, par conséquent, les tassements différentiels en surface.

d) poursuite de l'affaissement, ralenti au début, puis à vitesse constante sur les 5 années de mesures, avec cette fois un certain parallélisme de tous les points (après deux ans d'interruption les points de mesure se sont alignés en prolongement des courbes précédentes).

Pour les cinq points de nivellement représentés sur la figure 9 l'affaissement total mesuré est donc 350, 320, 180,145 et $100 \mathrm{~mm}$, à ajouter à ce qui s'est passé avant le début des mesures, qui est vraisemblablement du même ordre ou peut-être même supérieur; et comme la pente des courbes ne montre pas d'atténuation, le tassement tardif qui règne uniformément sur une zone étendue est susceptible de se poursuivre à l'échelle décennale, pouvant donc ajouter encore 100 ou $200 \mathrm{~mm}$ supplémentaires avant de se stabiliser.

Ainsi l'affaissement total maximal est-il de l'ordre du mètre, ou même davantage, ce qui correspond bien à la fermeture en profondeur de vides étendus de plus d'un mètre de hauteur restés ouverts en fin d'exploitation du charbon.

\section{3}

\section{Interprétation (cf. Fig. 10 et 11)}

Sitôt les affaissements localisés, une tentative de sauvetage par injections a été entreprise à l'automne 1981, mais à la suite des aggravations constatées un an plus tard, l'ordre de grandeur du traitement nécessaire est apparu considérable et a été considéré comme trop coûteux.

C'est alors que, dans le cadre d'une procédure d'appel, il a été recherché si le vide générateur était entièrement situé sous la parcelle du Petit Clos, ou bien s'étendait sous les parcelles contiguës restées propriété des Houillères. Il est possible en effet d'utiliser les schémas bien connus des affaissements miniers pour retrouver le vide générateur à partir de la cuvette des tassements en surface.

La cuvette dessinée sur la figure 10, comme celle de la figure 11, est allongée (en gros, suivant la rue LouiseMichel) et ses flancs sont nettement dissymétriques, celui sous les bâtiments du Petit Clos étant en pente plus douce que celui sous l'autre côté de la rue. Cette dissymétrie se justifie par le pendage général des couches de terrains, tant charbon que stériles.

Et c'est justement à cause de ce même pendage que la propagation des affaissements est déviée de la verticale pour se faire à peu près suivant une direction intermédiaire entre la verticale et la perpendiculaire aux couches, soit ici à 12 degrés environ sur la verticale (cf. Fig. 12). 


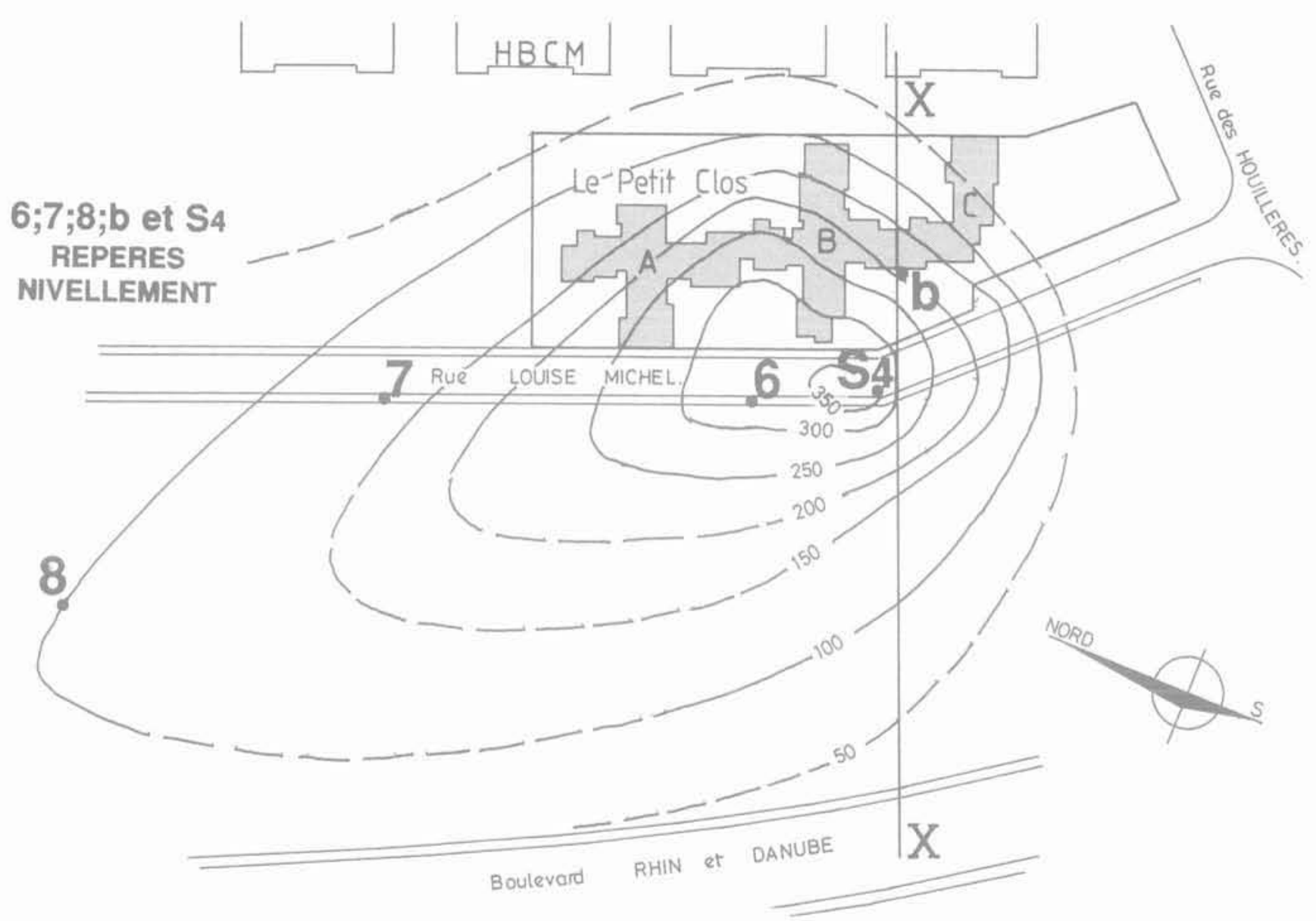

FG.11 Courbes d'iso-tassements entre janvier (ou avril) 1981 et juillet 1988. Iso-settlement curves between January 1981 and July 1988.

La comparaison des figures 10 et 11 montre la forte aggravation des tassements de surface entre octobre 1982 et juillet 1988 .

Comme le centre de la cuvette est déjà en limite de propriété, cette déviation conduit à placer le vide générateur en grande partie, et très vraisemblablement en totalité, en dehors de la parcelle du Petit Clos.

La proportion dépend en effet de la profondeur du vide, donc de la couche de charbon où il se trouve. Cette conclusion a toutefois été combattue par certains du fait qu'il existe sous le Petit Clos une ou deux failles susceptibles d'avoir modifié le schéma classique des cuvettes d'affaissements miniers.

\section{4}

\section{Sensibilité des constructions aux tassements}

Suivant leur taille, leur élancement, leur forme en plan et leurs matériaux constitutifs, les bâtiments sont plus ou moins sensibles aux tassements différentiels donc aux affaissements miniers. La cité HLM des HBCM voisine, rue de Chavassieux (cf. Fig. 7), est formée d'immeubles ramassés, relativement peu sensibles aux déformations. On ne sait s'ils ont ou non subi des affaissements mais il y a lieu de remarquer que leur tion par «chambres et piliers » par la faille dite de Sainte-Marie (cf. Fig. 14). Il a été reproché, à juste titre, aux immeubles du Petit Clos d'avoir des formes peu compatibles avec des affaissements même modérés, et notamment de ne pas avoir comporté entre eux d'espace. Toutefois, aucune restriction de ce type ne pesait sur la ZAC.

Dans les zones d'exploitation, il n'est pas impossible de construire et, d'ailleurs, les services spécialisés de Charbonnages de France relèvent des immeubles, pourvu qu'ils soient suffisamment monolithiques ou très bien chainés. Ce n'est d'ailleurs pas la descente des bâtiments qui constitue le phénomène le plus dangereux, lorsqu'elle est lente et uniforme, mais bien l'inclinaison et surtout les déformations horizontales du sol d'assise: sur le bord de la cuvette le terrain est en extension, par contre au cœeur de la cuvette il peut être en compression. Non seulement les bâtiments du Petit Clos ont été inclinés, les uns vers les autres, mais leurs fondations ont subi une extension.

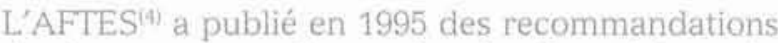
sur les tassements au-dessus des chantiers de tunnels, qui donnent un classement des désordres en fonction de l'ouverture e (en $\mathrm{mm}$ ) des fissures, d'après un consensus international:

\footnotetext{
19i Association française des travaux en souterrain.
} 


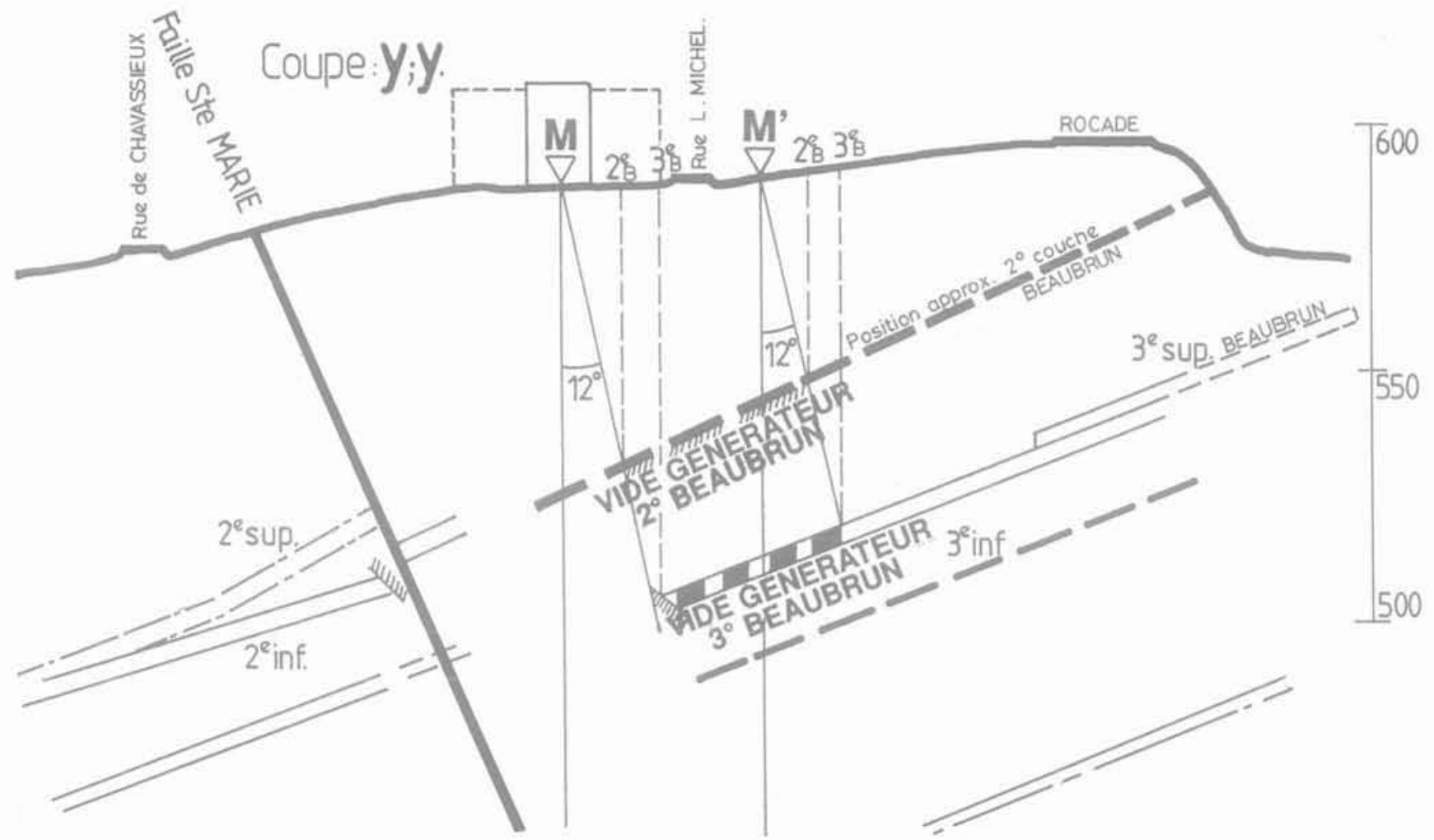

FIG. 12 Construction en coupe des vides générateurs à partir de la cuvette superficielle. Construction of generating voids (section).

$\begin{array}{lll}\text { classe 1: } & e<1 & \text { esthétiques } \\ \text { classe 2: } & e<5 & \text { esthétiques à traiter } \\ \text { classe 3: } & 5<e<15 & \text { fonctionnels } \\ \text { classe 4: } & 15<e<25 & \text { structuraux } \\ \text { classe 5: } & e>25 & \text { structuraux très graves }\end{array}$

Les fissures des bâtiments du Petit Clos entrent dans cette dernière classe; il n'est donc pas surprenant qu'ils aient dû être démolis.

\section{5}

\section{Feux de stériles et feux de mine}

\section{1}

\section{Feux de stériles}

Dans tous les pays charbonniers, les stériles déposés en remblais ou en terrils s'oxydent et s'échauffent. c'est banal; sur ces terrains la neige fond plus vite qu'au voisinage, et la végétation témoigne d'une température au-dessus de la moyenne (parfois on y fait deux récoltes par an); à l'intérieur ces remblais prennent par oxydation une couleur brique. C'est que les prétendus stériles ne le sont pas et que, loin d'être nulle, leur teneur en charbon permet une combustion qui peut aller jusqu'à la cuisson. Désormais devenu stable, le materiau obtenu est apprécié pour divers usages, notamment les chaussées routières.

En Allemagne, en Belqique et aussi en France, certains terrils ont même été entièrement repris par des entre- prises spécialisées pour extraire et brûler dans les centrales thermiques le charbon encore contenu (jusquà 12 ou $14 \%$ ). Ce qui n'est pas réutilisable est remis en dépôt et végétalisé (terril du Quesnoy près de Mons, Belgique).

La combustion de ( stériles » est un phénomène banal dans la région stéphanoise, et des fumerolles sont attestées en maints endroits sur une carte de 1969 destinée à l'aménagement du territoire.

Lorsque les températures et les gaz de combustion sont jugés nuisibles, les terrils sont traités par embouage, c'est-à-dire injection d'un mélange d'eau et de cendres de centrale thermique. Un tel traitement a été appliqué (voir ci-dessus) à la zone du boulevard Rhin et Danube, en liaison avec la Ville, et avec l'autorisation de la DEE.

\section{2}

\section{Feux de mine à Côte Chaude}

Quant aux feux de mine, ils affectent aussi bien le charbon des couches en cours d'exploitation, que celui des "vieux travaux ». C'est bien de tels feux qu'il s'agit dès lors que des sondages ont confirmé en 1981 des hautes températures en profondeur, $240^{\circ} \mathrm{C}$ à $35-45 \mathrm{~m}$; les trois sondages de la Vallée de l'Ondaine (Fig. 8) placent les points chauds suivant le pendage de la deuxième couche Beaubrun; au Petit Clos on a mesuré de $100^{\circ} \mathrm{C}$ à $280^{\circ} \mathrm{C}$ mais toujours sur la même couche, il ne s'agit donc pas de stériles.

Pour le Laboratoire central des ponts et chaussées en septembre 1981, al'existence de feux de mine anciens ne fait pas de doute $)$. 


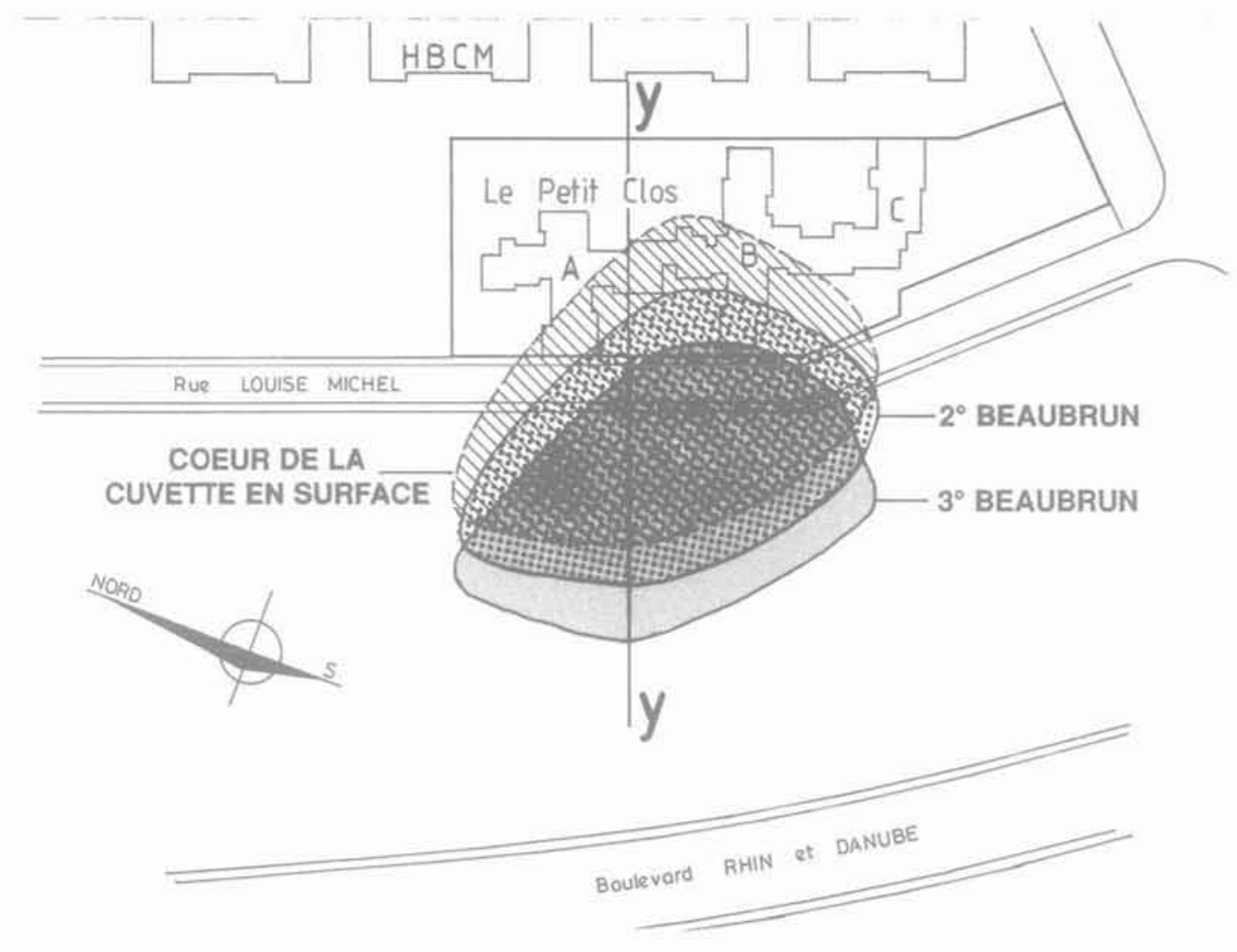

FIG.13 Localisation des vides générateurs en plan. Localization of generating voids (plan view).

L'origine en est incertaine, mais parmi les hypothèses, il y a la remontée de la nappe après fermeture des exploitations profondes, qui a pu brasser l'air. Sous le site, la nappe est vers la cote 518 (grâce au pompage dans le puits Couriot), soit à $70 \mathrm{~m}$ de profondeur, donc plus bas que les sondages et les feux. Les Houillères ont d'abord niẻ le feu car les fumerolles ne contenaient guère d'oxyde de carbone, mais il a été finalement confirmé par le CERCHAR (Centre d'études et de recherches des Charbonnages de France).

Une reconnaissance locale en 1988 (par la Compagnie de prospection géophysique française) a mis en évidence des anomalies chaudes $\left(+4^{\circ} \mathrm{C}\right)$ le long de la rue Louise-Michel et sur une ligne perpendiculaire passant entre les bâtiments $A$ et $B$, situant le pic de chaleur aux environs du centre de la cuvette d'affaissement.

Une thermographie régionale effectuée par l'Institut géographique national fait ressortir l'affleurement de la deuxième Beaubrun le long du boulevard Rhin et Danube.

\section{3}

\section{Les connaissances sur les feux de mine}

Les publications sur les feux de mine sont rares, au point que l'entrée n'existe pas à la bibliothèque de l'École des mines de Paris; il faut chercher «Aérage $»$, parce que les feux de mine troublent les circuits d'aérage (Froger et al., 1975). Un souci permanent de d'asphyxie, de brûlures et d'explosion au contact du grisou.

Un document de synthèse a été établi en 1987 (Vielledent), Mémento sur les feux et incendies dans les exploitations souterraines. On peut noter que la lutte contre les feux n'est envisagée que dans le cadre de l'exploitation; il n'y aurait pas de cas en France concernant des tiers; et il n'est fait aucune allusion à SaintÉtienne.

Hors des mines, les travaux souterrains affectant des terrains combustibles peuvent aussi allumer des feux; ainsi une couche de lignite s'est-elle enflammée à Neuilly lors du creusement du RER (Réseau Express Régional) (outre les émanations dans les caves, un immeuble aurait souffert de tassements).

Un rapport du Bureau of Mines des États-Unis (1983) étudie de façon détaillée le cas de la mine de Centralia, dans une région (la Pennsylvanie) où les feux sont nombreux et difficiles à maitriser: le feu décrit a débuté en 1962 dans un dépôt de stériles au contact de l'affleurement de la couche; il a progressé le long de la couche, de $150 \mathrm{~m}$ en 11 mois (soit $50 \mathrm{~cm}$ par jour en moyenne) jusque sous la petite ville où divers bầtiments ont dû être évacués à cause des gaz toxiques ou d'une chaleur excessive; après vingt ans de lutte (et une dépense de plus de 20 MF) le feu n'a pu être stoppé; on envisage l'extraction complète de la couche synclinale (pour 500 MF).

Les ouvrages anciens sur les mines de charbon, et notamment sur la région stéphanoise sont prolixes: divers ouvrages historiques (de Bournon, 1784 ; Simo- 


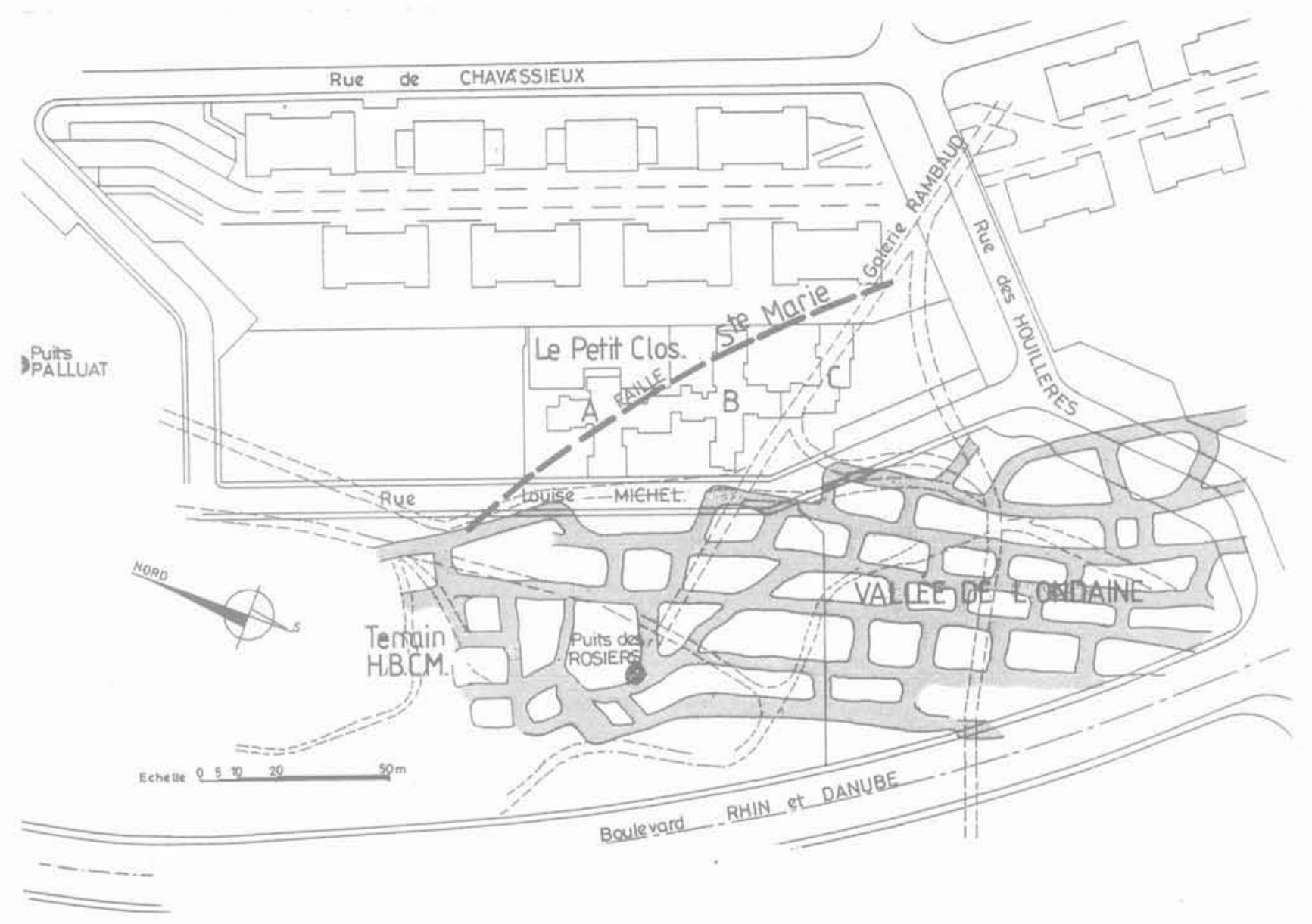

FIG.14 Zone d'exploitation de la couche troisième Beaubrun par «chambres et piliers ».

Exploitation of third Beaubrun layer using « room and pillar».

nin, 1867 et Leseure, 1901) établissent à la fois le caractère banal des feux de mine dans le bassin stéphanois et leur grande ancienneté :

- dès 1605 «Saint-Étienne est empestée de l'importune et sale fumée des trois montagnes incessamment brúlantes, flamboyantes et embrasées, la Mina, la Valla, la Bouta );

- de même, à la Ricamarie (en 1784) “la mine brûle depuis plus de 300 ans $)$;

- et en 1765 «la plus grande partie du territoire (...) est menacée de ruine, comme il appert par deux montagnes qui brûlent depuis plusieurs années »).

Simonin déclare que les feux se produisent spontanément, en raison de la décomposition ou fermentation du charbon, "surtout s'il est sous forme de menus morceaux ; et il incrimine particulièrement « les vieux travaux, ces vides irréguliers existant dans presque toutes les mines. Ce sont les plus mauvais voisins d'une houillère; ils sont le théâtre d'incendies souterrains ».

Depuis la fermeture des mines les feux se sont plusieurs fois rappelés aux Stéphanois : ainsi la construction du marché de gros en 1971 a allumé un feu qui a été maîtrisé par embouage; et tout récemment, lors de l'aménagement du puits Couriot en musée de la mine en 1988, une couche "Beaubrun » horizontale à $10 \mathrm{~m}$ sous le (carreau ) a pris feu à l'occasion d'un modeste terrassement en surface, Les documents établis pour les besoins de I'urbanisme (cartes synthétiques des exploitations passées, en 1969, mises à jour en 1981, par
Épures, puis un atlas de 1986-1987), mentionnent notamment les fumerolles ainsi que les couches de charbon à moins de $50 \mathrm{~m}$ de profondeur.

Revenons aux sourches modernes déjà citées, tant françaises qu'américaines (toutes postérieures aux faits cités ici), elles s'accordent sur les points suivants: a) La combustion spontanée fait suite à l'adsorption d'oxygène et parfois de vapeur d'eau, qui est exothermique. Si l'apport d'air ne suffit pas à évacuer la chaleur produite, la température s'élève petit à petit; à partir de $75^{\circ} \mathrm{C}$ à $80^{\circ} \mathrm{C}$ le phénomène devient irréversible, l'oxydation puis la pyrolyse sont toujours exothermiques, la température s'élève de plus en plus vite et, au-delà de $850^{\circ} \mathrm{C}$, il y a combustion franche. La phase d'échauffement peut durer des semaines ou des mois, mais l'accélération finale ne dure que quelques jours ou quelques heures.

La largeur critique d'un massif sujet à feu est comprise entre 5 et $25 \mathrm{~m}$; au-dessous la circulation d'air limite l'échauffement, au-dessus elle est insuffisante pour alimenter l'oxydation. Le feu progresse en remontant le courant d'air.

b) Certains charbons sont plus susceptibles que d'autres (sans que les facteurs en soient clairement identifiés: porosité, fragmentation, teneur en pyrite) : certaines couches susceptibles sont rapidement repérées (à Saint-Étienne les couches «Beaubrun » sont appelées les ( brûlantes $»)$; diverses conditions locales sont énumérées en sus, par exemple le voisinage d'une 


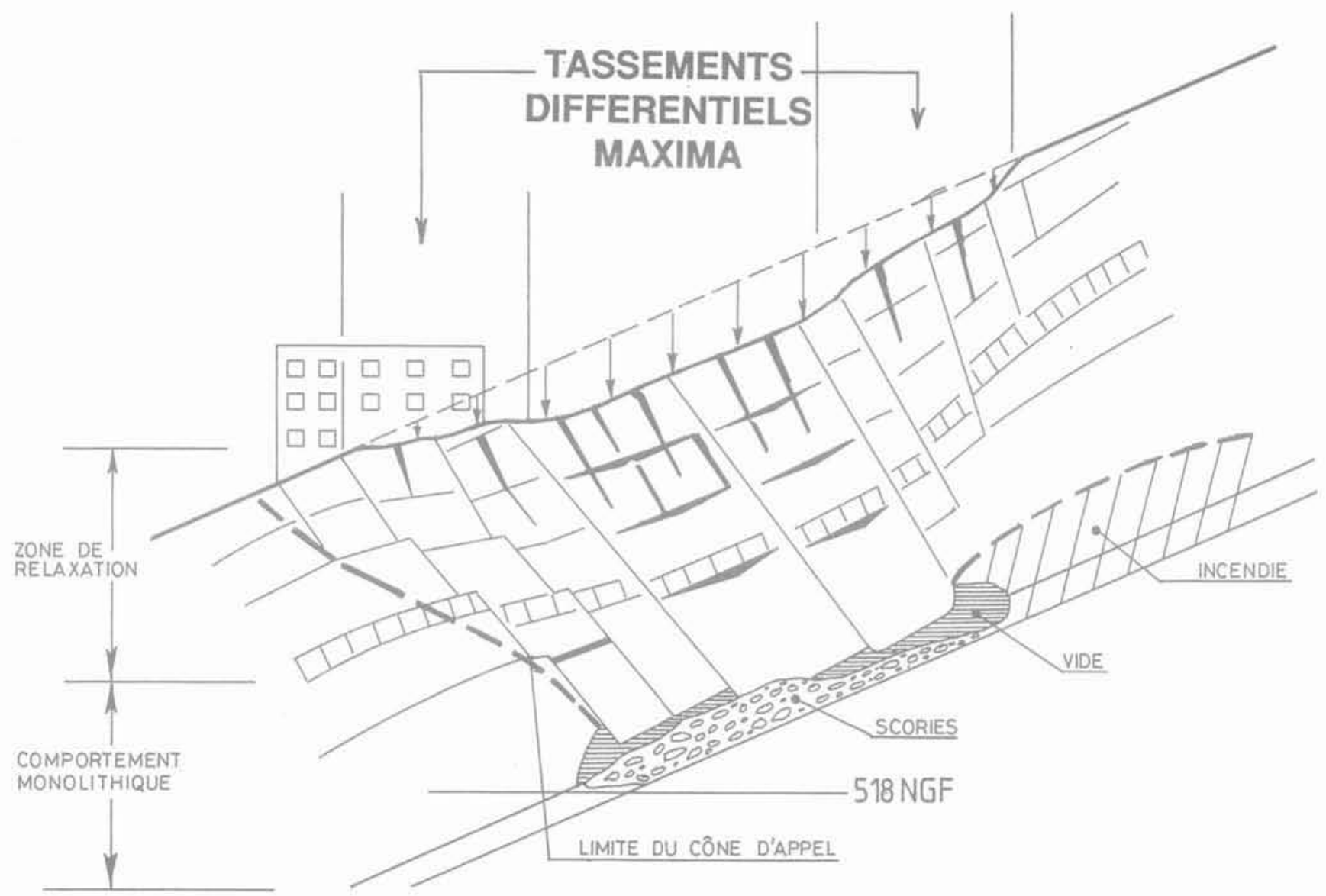

FIG.15 Coupe interprétative Bachy du 4 juin 1981. Interpretative Bachy section in 1981.

faille, ou des conséquences de l'exploitation, par exemple le foudroyage ou simplement un pilier écrasé. c) La conductivité thermique des terrains étant faible, la chaleur dégagée ne se propage pas loin, mais la durée de refroidissement est très grande, quelques années pour un petit feu, un siècle ou davantage pour un plus grand.

d) La propagation de la chaleur s'accompagne de dilatation ou de contraintes, susceptibles de provoquer des ruptures.

Le cas des ( vieux travaux » n'est traité explicitement qu'aux États-Unis: les feux dans les anciennes mines y sont fréquents, ils menacent la surface d'émanations toxiques, d'affaissements et de températures excessives ; en particulier des feux inaccessibles se développent dans les zones foudroyées, qui se rallument très souvent, même après des années.

\section{4}

\section{La lutte contre les feux}

Dans les mines en exploitation le premier souci est d'isoler le quartier en feu et de boucher toute entrée d'air; en effet l'eau n'est pas un remède suffisant, elle est même susceptible d'accélérer la réaction. Et selon le Bureau of Mines, l'embouage en mine n'est efficace qu'à $50 \%$, moins encore dans les couches inclinées. La loi de refroidissement en fonction du temps montre que les hautes températures subsistent très longtemps malgré l'embouage; or dès $75^{\circ} \mathrm{C}$ un feu peut se rallumer spontanément à tout instant. Il n'est donc pas étonnant que les diverses tentatives d'embouage réalisées par les HBCM en 1981-1982 et 1983 n'aient pas donné à Côte Chaude les résultats escomptés.

\section{6}

\section{Conclusions}

La cause du sinistre du Petit Clos à Saint-Étienne est un affaissement de la surface du sol, imputable à la fermeture tardive de vides résiduels hérités d'exploitations minières anciennes (plus d'un siècle, peut-être deux). L'affaissement s'est manifesté pendant la construction des trois bâtiments sinistrés, avec des périodes de calme et d'accélération et s'est poursuivi pendant dix ans au moins.

A notre avis, il est exclu que les travaux de construction aient déclenché l'affaissement, qui est d'ailleurs sensiblement plus étendu que l'emprise des bâtiments (cf. Fig. 10 et 11).

Les affaissements miniers au-dessus des exploitations en cours en sont des conséquences bien connues et en général maitrisées; pour être plus rares, les affaissements tardifs ne sont pas inconnus, ils affectent plutôt des " stots » supposés stables à long terme; mais l'influence des feux est hélas beaucoup moins connue il y a très peu de documentation sur les feux (comme sur les conséquences dommageables rares de beau- 
coup d'industries); le grand public et même les autorités locales de la Ville et de l'Equipement les ignorent; et la DRIRE ${ }^{(5)}$, qui les connaît, n'est pas consultée sur le projet de ZAC.

On ignore comment le feu a influencé l'affaissement, mais la présence d'un feu est établie. Ce feu est une suite tardive de l'exploitation mais son implantation exacte est incertaine ; il est à l'échelle de la ZAC plutôt que de la parcelle, à l'échelle du siècle plutôt que de l'année ou de la décennie; il pourrait être lié à la galerie Rambaud; il ne pouvait guère être ignoré des Houillères, et il parait extraordinaire qu'aucune autorité stéphanoise ne se soit préoccupée de cette éventualité.

L'affaissement de surface est partagé entre le soussol du Petit Clos et celui de son voisin, les HBCM le feu aussi. La clause d'exonération paraît évidemment léonine tant que l'acheteur n'a pas de compétence en exploitation minière. En première instance le demandeur a été débouté en raison de cette clause, et le jugement a été confirmé en appel, bien que le vide générateur de l'affaissement soit pour l'essentiel chez le voisin (on sait qu'il n'y a pas de vice caché entre professionnels avertis, or l'acheteur était un ancien professionnel de la Mine)

Les vides datent d'une époque antérieure à la dernière concession, ils sont donc sans rapport avec l'activité actuelle des Houillères de la Loire.

L'architecte du Petit Clos a fait confiance à son maître d'ouvrage, ce n'est pas surprenant puisqu'ancien des Houillères, celui-ci devait être au courant des risques. L'entrepreneur n'a rien à se reprocher dès lors que les désordres ne sont pas dus à des erreurs ou fautes de sa part (sauf à constater des dénivellations en cours de travaux, il est curieux qu'il n'ait rien soupçonné). Le bureau de contrôle ne s'est douté de rien. Tous peuvent apparaître bien légers pour avoir accepté des bâtiments dont les formes individuelies et les liaisons étaient très sensibles aux tassements différentiels, toujours susceptibles de se produire à plus ou moins long terme en site minier.

Mais les autorités concernées par l'aménagement de la ZAC partagent la légèreté première d'avoir laissé vendre et construire sans se donner des moyens de surveillance et sans demander aux acheteurs de prendre des précautions particulières alors que les

airection régionale de IIndustrie, de la Recherche et de l'Environnement (qui a repris notamment les attributions de l'ancien Service des mines).

affaissements et les feux ont déjà donné lieu à bien des litiges à Saint-Étienne. Que l'on doive raser des bâtiments qui viennent d'être construits et que le terrain d'une ZAC se soit avéré à l'usage inconstructible, c'est un comble!

\section{7}

\section{Chronologie}

25 janvier 1973

29 janvier 1974

14 février 1975

1979 à 1980

Décembre 1979

Mai 1980

Septembre 1980

1980-1981

Début 1981

Mai-juin 1981

4 juin 1981

14septembre 1981 Rapport Laboratoire Central des

Nov,-déc, 1981

Début 1982

Avril 1982

Décembre 1982

22 décembre 1982

13 janvier 1983

Avril-mai 1983

14 mai 1983

Juin 1983

2 juin 1983

13 octobre 1983

8 octobre 1985

17 février 1989

1989

6 février 1992

1993

Arrêté préfectoral créant la ZAC Plan d'aménagement de zone

Le Petit Clos, étude géotechnique Sageor

Construction des bâtiments A, B, C

Livraison du bâtiment $\mathrm{C}$

Livraison du bâtiment $B$; premières fissures du bâtiment $C$

Livraison du bâtiment $\mathrm{A}$

Embouages HBCM

Début des nivellements

Sondages profonds Bachy

Rapport Bachy

Ponts et Chaussées

Injections de consolidation par

Bachy

Expertise Filliat pour TGI SaintÉtienne

Embouages HBCM

Reprise des affaissements

2 Rapport Simecsol

Rapport Simecsol

Embouages HBCM et accélaration brutale des affaissements

Arrêt des embouages prescrit par G. Filliat

Arrèté de péril, évacuation totale des bâtiments A, B et C

Dépôt rapport Filliat

Rapport Simecsol

Jugement TGI Saint-Etienne

Rapport CERCHAR

Rapport Duffaut-Bardot pour cour d'appel de Lyon

Arrèt cour d'appel de Lyon

Démolition totale des bâtiments A, B et C

\section{Bibliographie}

Bournon (de) - Lithologie des environs de Saint-Étienne, 1784.

Chaiken R.F., Brennan R.J., Heisey B.S., Kim A.G., Malenka W.T, Schimmel J.T, - « Problems in the Control of Anthracite Mine Fires: A Case Study of the Centralia Mine Fire (August 1980) D, Bureau of Mines Report of Investigations, rapport RI 8799/1983.

Duvauchelle C. - « Mécanismes d'évolution des cavités souterraines n, Mémoire d'Ingénieur CNAM, 1985.

Froger C., Jeger C., Pregerman S, - Aérage (chap. 3 «Feux de mine»), Document
SIM N3, Industrie Minérale 2, p. 54-70, 1976.

Gray R.E., Bruhn R.X., Turka R.J. - "Study and Analysis of Surface Subsidence over the Mined Pittsburgh Coalbed $⿻$, Final Report, US Bureau of Mines, Pittsburgh, PA, 1977.

Grüner - Description détaillée du Bassin de la Loire, 1882.

Josien J.-P. - "Carrières souterraines abandonnées, diagnostic et caractérisation des aléas, Bulletin AIGI 51, 100, 1995.

Leseure - Historique des mines de houille du département de la Loire, 1901.
Shadbolt C.H. - «Mining Subsidence. Historical Review and State of the Art, Large Ground Movements and Structures, Proceedings of Conference at University of Wales, Cardiff, James D. Geddes ed. New York, John Wiley and Sons, 1978.

Simonin - La vie souterraine, Paris, Hachette, 1867.

Vielledent L. - « Mémento sur les feux et incendies dans les exploitations souterraines », Industrie Minérale, Les Techniques 4, 67 pages, 1987 .

Vidal V. - Cours d'exploitation des mines, Paris, Dunod, 1961. 